\title{
Is there any new parameter as difficult airway predictor in TMJ disorders?
}

Tapia B*, del Castillo J.L**, Garrido E*, De Latorre J*, Gilsanz F* *Anesthesiology **Maxillofacial Surgery Department La Paz University Hospital. Madrid.

Spain

\section{BACKGROUND AND GOAL OF STUDY:}

Temporomandibular joint disorders (TMJD) :articular cartilage, disc, synovium and subcondral bone deterioration. Wilke's stages: classification system to categorized the progressive symptoms of TJMD. These patients usually have a interincisor distance $<3,8 \mathrm{~cm}$, a difficult airway (DA) predictor. The aims were to describe the incidence of DA and trying to find others DA predictors in this patients.

\section{MATERIALS AND METHODS}

Observational study, after ethic committee approval, $120 \mathrm{TMJ}$ disorder patients were included. There were recorded demographic data, airway predictors, MRI and arthroscopic finding and airway management data.

\section{RESULTS AND DISCUSSION}

No differences in demographic data., Only in gender) Clinical symptoms weren $t$ DA predictors Neither MRI findings or arthroscopic findings could be considered DA predictors

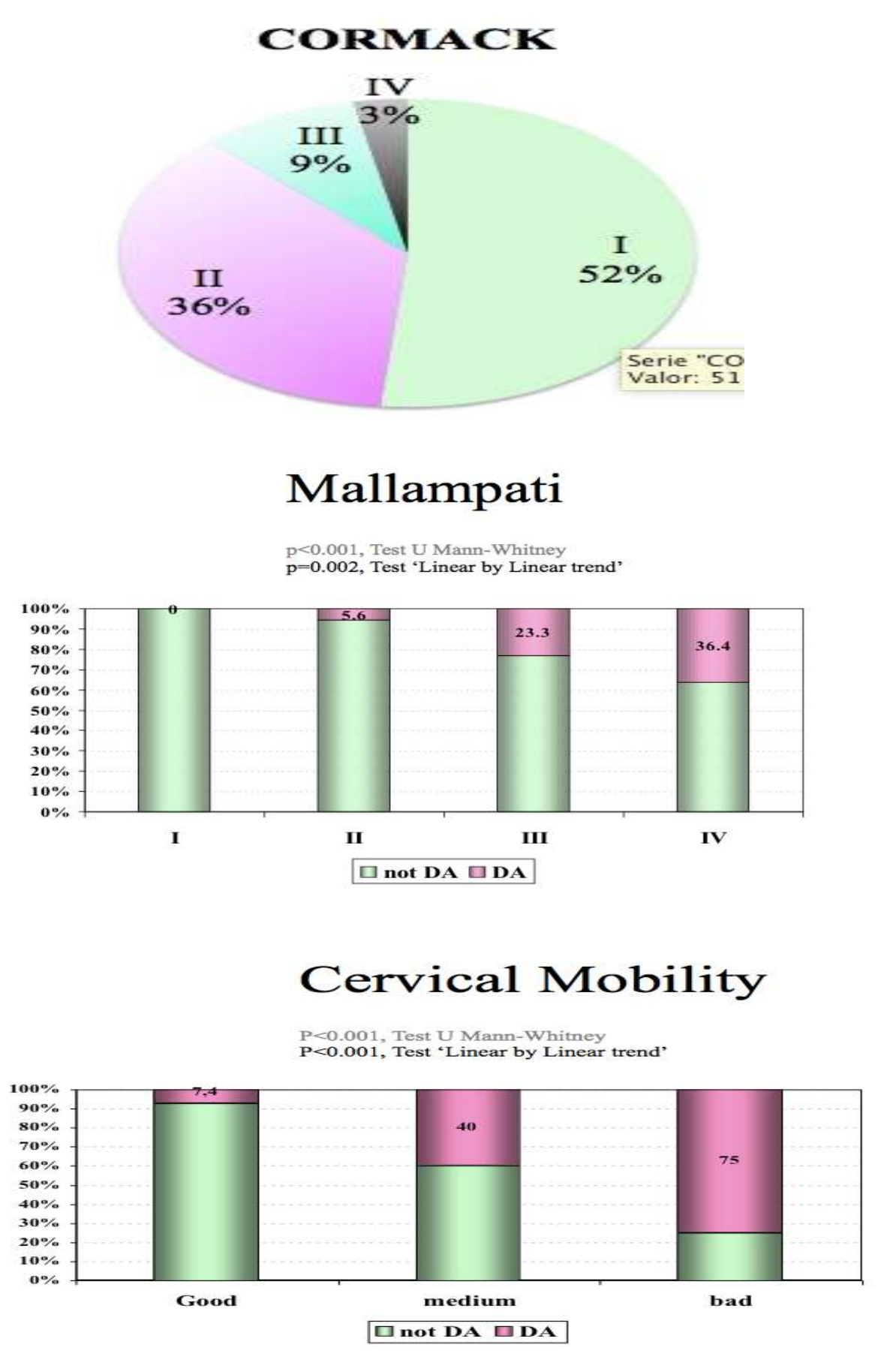

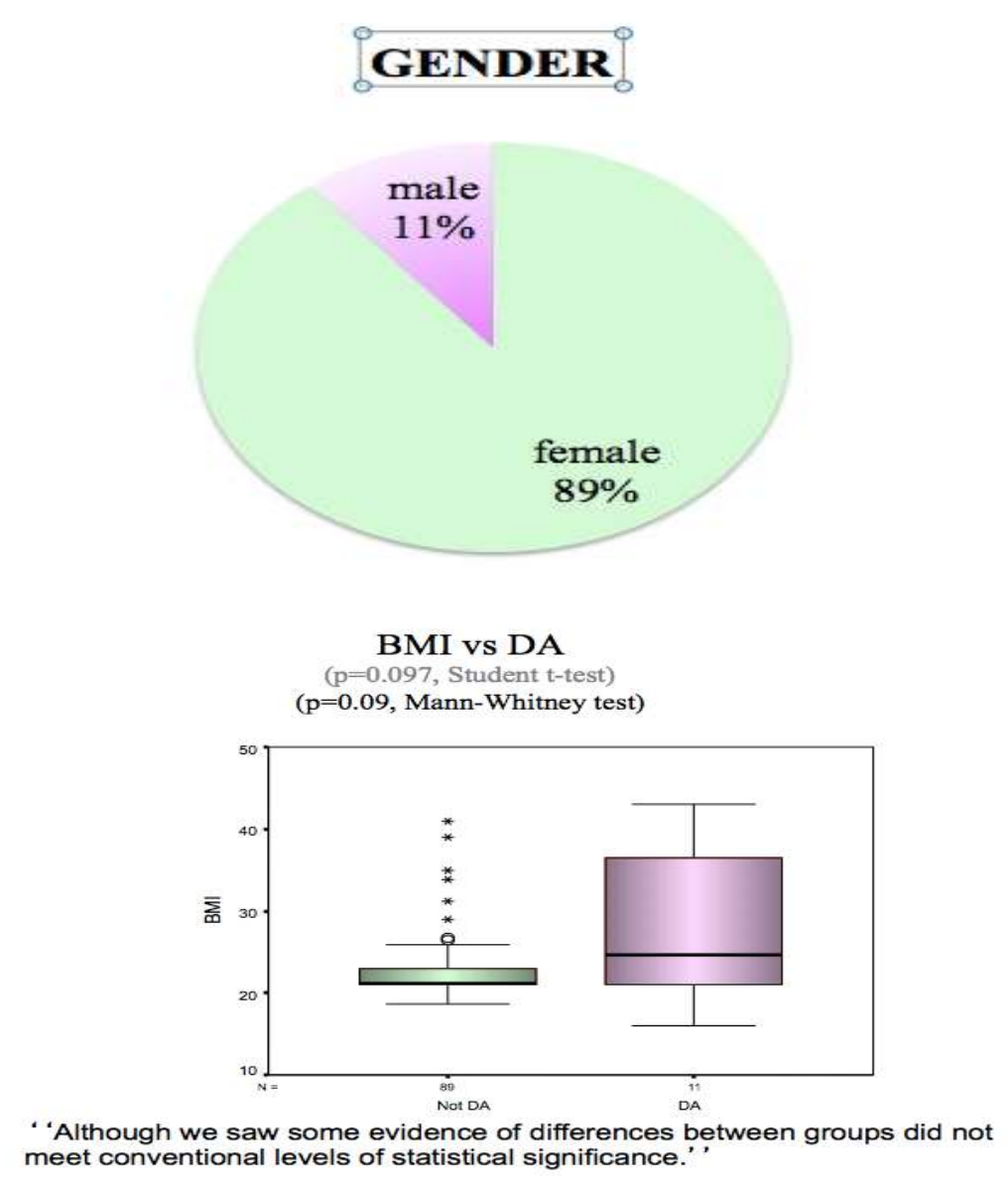

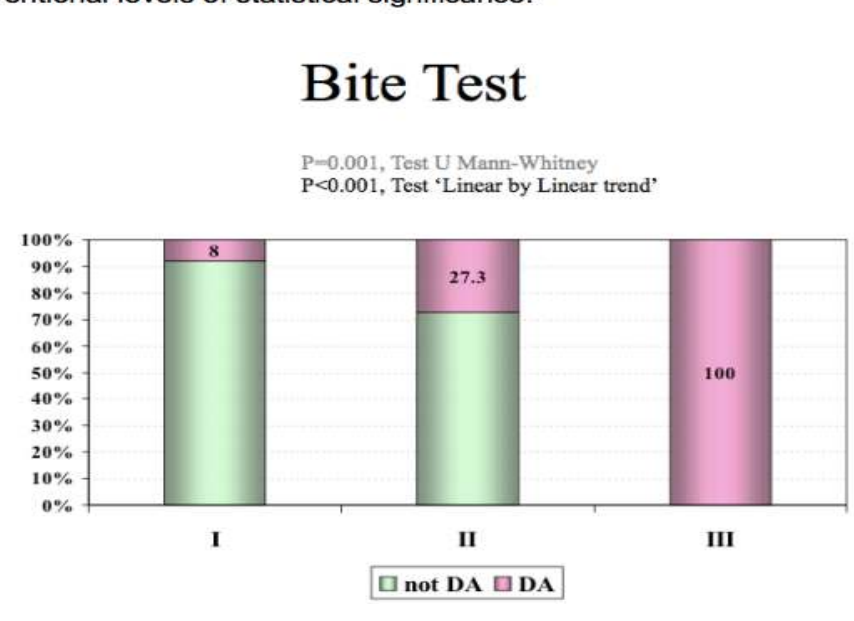

\begin{tabular}{|l|l|}
\hline Easily ventilate & $100 \%$ \\
\hline FBC & $3 \%$ \\
\hline Burp/ VDL & $13,5 \%$
\end{tabular}

WILKE'S

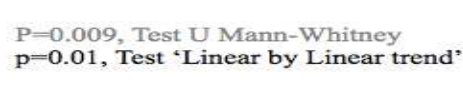

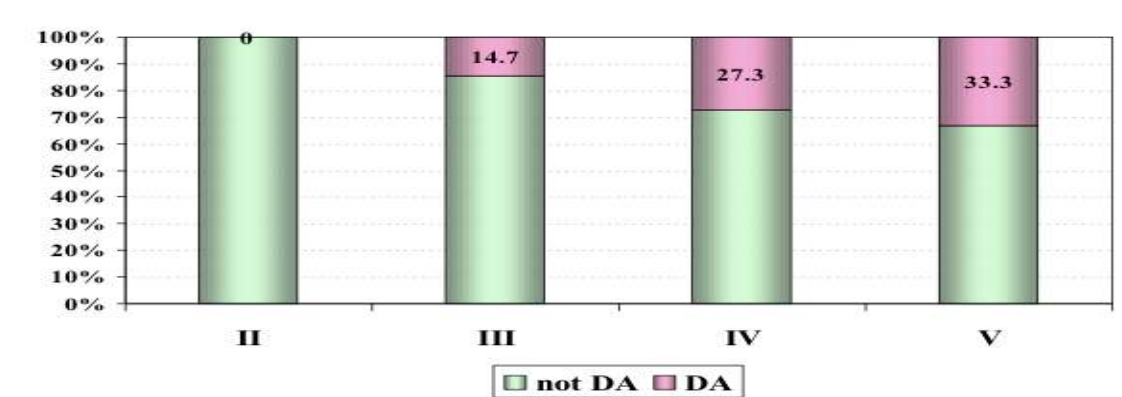

WILKE'S STAGES

\begin{tabular}{|c|l|}
\hline STAGE & CLINICAL \\
\hline I & Clicking during opening. No pain \\
\hline$\|$ & Pain tenderness, headache, Clicking and occasional locking \\
\hline II & Pain, major mechanical symptoms \\
\hline IV & Reduced motion, chronic symptoms \\
\hline V & Decreased motion, reduced function \\
\hline
\end{tabular}

CONCLUSION

TMJ disorders patients have an easy airway management and can easily be intubated with conventional laryngoscopy. MRI and clinical symptoms aren't good DA predictors.

As previous studies showed, Mallampati score, BMI, cervical mobility and bite test are good predictors. We can add, as new DA predictor, Wilke's stages

\section{REFERENCES}

